\title{
Substantia nigra hyperechogenicity in Polish patients with Parkinson's disease
}

\author{
W. Ambrosius, S. Michalak, M. Owecki, M. Łukasik, J. Florczak-Wyspiańska, W. Kozubski \\ Department of Neurology, Poznan University of Medical Sciences, Poznan, Poland
}

[Received 24 February 2014; Accepted 28 March 2014]

Background: Hyperechogenicity of the substantia nigra (SN) measured by transcranial sonography (TCS) is a characteristic feature observed in patients with Parkinson's disease (PD). To our knowledge, no SN hyperechogenicity data are available for Polish population. Moreover most of studies come from few centres, which used the one type of ultrasound device. The main aim of the study was to investigate the association between PD and SN hyperechogenicity measured by sonographic machine, not assessed so far.

Materials and methods: In this study cross-sectional study SN hyperechogenicity was evaluated in 102 PD patients and 95 control subjects. Midbrain was visualised by Aloka Prosound 7 ultrasound device. SN area measurement, the relation to the clinical features of $P D$, inter- and intra-observer reliability were evaluated. Results: We confirmed that SN echogenicity is significantly increased in PD patients compared to control subjects $(p<0.001)$. The area under curve for PD patients vs. controls was 0.93. Receiver operating characteristic analysis indicated a cut-offs for SN echogenicity at $0.19 \mathrm{~cm}^{2}$ with accuracy equal to $90 \%$, specificity - $86 \%$ and sensitivity - 93.7\%. The SN hyperechogenicity was not related to PD clinical findings. Reliability was good if an experienced sonographer performed the $S N$ measurements.

Conclusions: This study shows that the SN abnormality observed by TCS is a specific feature, which can be helpful in the process of PD diagnosing. (Folia Morphol 2014; 73, 3: 267-271)

Key words: Parkinson disease, substantia nigra, transcranial sonography

\section{INTRODUCTION}

Parkinson's disease (PD) is one of the most frequent neurological conditions $[13,23]$ and some proved that it is the second most common neurodegenerative disorder [15]. Despite the seriousness of the issue the one specific test which might help to put final diagnose has not been established; dopamine transporter single photon emission computed tomography brain imaging (DaT-SPECT) which could be useful in the assessment of parkinsonism with nigrostratial degeneration is expensive and not widely available. In the routine practice the clinical symptoms and criteria still decide about the diagnosis of the disease.

Transcranial brain sonography (TCS) is a relatively new imaging technique that can reveal selected structures of the brain parenchyma. Several years ago changes of the substantia nigra (SN) echogenicity in TCS in PD patients were revealed [2]. Recently many 


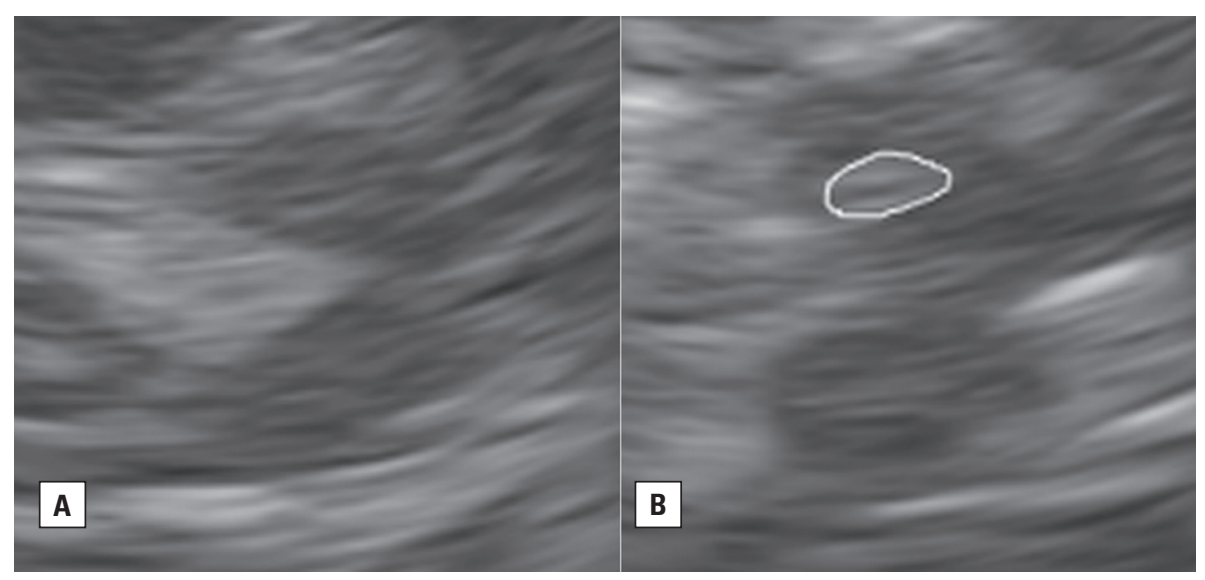

Figure 1. Ultrasound image of midbrain with; A. Substantia nigra (SN) in control subject; B. SN in Parkinson's disease patient (circled by white line).

studies have confirmed that SN hyperechogenicity (Fig. 1) is distinguishing feature of PD [4, 25, 32], moreover some of them have shown that this phenomenon is observed in about $90 \%$ of PD cases [11, 20]: it seems that this non-invasive and low-cost imaging technique, may be valuable in PD diagnosing process. However we should emphasize that large observations have been done on one type of the ultrasound system (Siemens Sonoline, Germany) and experts in the field claim that every laboratory should establish its own specific reference values for SN echogenicity measurements $[6,29]$.

To our knowledge, the SN hyperechogenicity visualised by TCS have not been assessed in Polish population.

The aim of our study was to analyse this sonographic phenomenon in the Polish PD population and control subjects using a different (until now not evaluated) ultrasound device (Aloka Prosound, Japan). In this trial the relation of the sonographic findings to the clinical features of PD patients was also evaluated. Additionally inter- and intra-observer reliability was calculated.

\section{MATERIALS AND METHODS}

In this cross-sectional, case-control study 102 consecutive patients with sporadic type of PD hospitalised between August, 2011 and April, 2013 at the Neurology Department in the university hospital in Poznan (Poland) have been included. The local Ethics Committee of the Poznan University of Medical Sciences approved the protocol of the study.

The PD was diagnosed in the agreement with given criteria, which consist of the presence of 3 (pro- bable PD) of the 4 cardinal features (resting tremor, bradykinesia, rigidity, asymmetric onset), and with significant reaction to conventional therapy (L-dopa or a dopamine agonist) [16]. All patients underwent magnetic resonance imaging in order to exclude symptomatic parkinsonism. None of the PD cases has recognisable cause of secondary parkinsonism or presented atypical symptoms such as pyramidal signs, gaze palsy or cerebellar ataxia.

The control group consisted of 95 patients without any central nervous disease. They were consecutively hospitalised because of musculoskeletal disorders and polyneuropathies.

All subjects underwent comprehensive clinical and TCS examination, which were performed independently by physicians blinded to the results of the other investigation. To assess the disease severity, the PD patients were examined using the Unified Parkinson's Disease Rating Scale (MDS-UPDRS) and Hoehn and Yahr stage grading [17, 19]. The categorisation of the patients with regard to their motor symptoms was performed using given criteria according to UPDRS III items specific for bradykinesia, rigidity and tremor. Patients were considered to have a tremor-dominant type (TD) if their symptom score achieved 3 points in tremor items, and score 0 or 1 in bradykinesia and rigidity items. The subjects were classified to have an akinetic-rigid type (AR) if their symptom score was 3 points in 3 bradykinesia and rigidity items, and a score of 0 or 1 in tremor items. The mixed type has been chosen if patients had another combination of scores specific for bradykinesia, rigidity, and tremor [22].

TCS examination was performed as described in experts' recommendations and review article [1,30]. 
Briefly, an ultrasound device with a phased array 2.5-3.5 MHz transducer (Aloka Prosound, Japan), the penetration depth $14-16 \mathrm{~cm}$, automatic adjusted gain compensation and brightness and examined scanning plane through the temporal acoustic bone window have been used. On the optimal images of the butterfly-shaped hypoechogenic midbrain surrounded by hyperechogenic basal cisterns we manually encircled the echogenic area corresponding to the $\mathrm{SN}$. An expert sonographer examined all subjects and he performed measurements twice offline. Additionally, experienced sonographer but with only basic skills in the TCS repeated measurements in randomly selected 30 PD patients and 30 control subjects. Thus intra-rater and inter-rater reliability could be estimated.

\section{Statistical analyses}

Descriptive statistics are given as mean and standard deviation (SD). Normal distribution of data was assessed by Shapiro-Wilk test. In the statistical analysis, nonparametric test (Mann-Whitney $\mathrm{U}$ test), parametric unpaired $t$ test, Fisher's exact test have been applied. Receiver operating characteristics (ROC) were performed to assess the accuracy of TCS for differentiation of PD patients and control subjects.

For inter-rater and intra-rater correlation, intra-class correlation coefficients (ICC, two-way random model, absolute agreement) for $\mathrm{SN}$ hyperechogenicity measurements were determined. Reliability values were classified as: $0.00-0.10-$ none; $0.11-0.40-$ slight; $0.41-0.60$ - fair; $0.61-0.80$ - moderate; 0.81-1.00 - substantial [27].

Differences were assumed to be significant at $p<0.05$. Statistical evaluations were conducted using MedCalc Software (version 12.7.2, Ostend, Belgium).

\section{RESULTS}

Seven $(7 \%)$ subjects were excluded from the study PD group due to an insufficient temporal bone window. Final statistical analysis included 95 PD patients and 95 controls. There was no significant difference in sex and age between the groups.

The mean area of SN hyperechogenicity was significantly greater in the PD patients than in the controls $0.23 \pm 0.04 \mathrm{~cm}^{2}$ and $0.12 \pm 0.06 \mathrm{~cm}^{2}$, respectively, $\mathrm{p}<0.001$ (Fig. 2).

ROC of SN echogenicity showed an area under the curve of 0.93 (95\% confidence interval [CI], 0.89-0.96) for differentiation of PD patients and control subjects. Analysis of ROC indicated a cut-off for SN

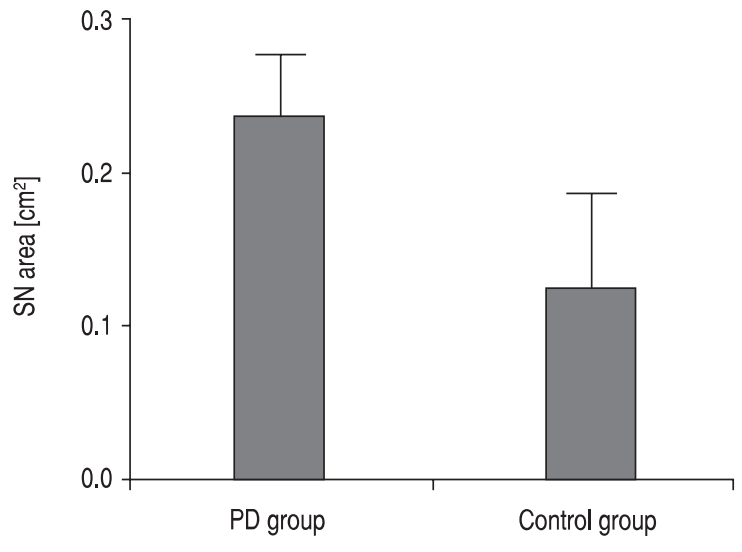

Figure 2. Area of substantia nigra (SN) hyperechogenicity in Parkinson's disease (PD) patients and control subjects (box plots show mean with standard deviation values).

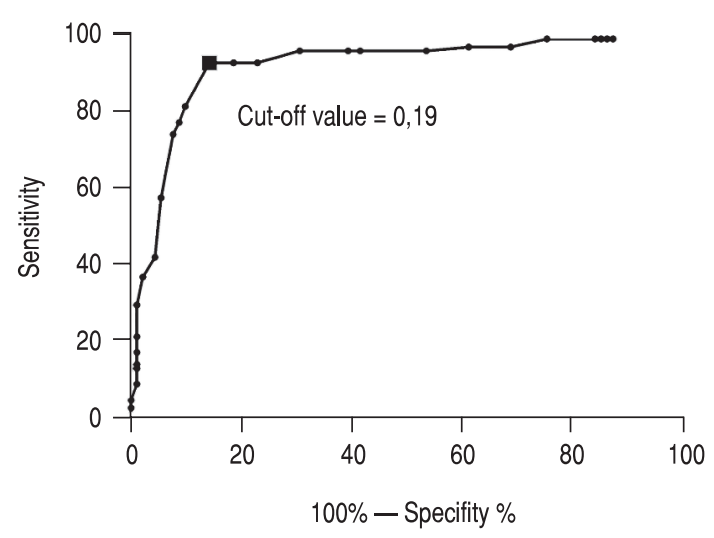

Figure 3. Receiver operating characteristic curve for discrimination of Parkinson's disease patients $(n=95)$ and control subjects $(n=95)$ by sonographic measurement of substantia nigra echogenicity; for cut-off value $=0.19 \mathrm{~cm}^{2}$, sensitivity is $93.7 \%$ and specificity is $86 \%$.

echogenicity at $0.19 \mathrm{~cm}^{2}$ (Fig. 3). For this cut off accuracy was $90 \%$, specificity was $86 \%$, sensitivity was $93.7 \%$, the positive predictive value was $85.7 \%$ and the negative predictive value was $82.8 \%$. Seventy eight $(82 \%)$ of PD patients and $10(10 \%)$ of control subject had SN echogenicity above cut-off value i.e. $>0.19 \mathrm{~cm}^{2}$.

The intra-observer ICC value for experienced sonographer was very good -0.94 (95\% Cl 0.86-0.97), for physician with basic skills in the TCS was moderate - 0,79 (95\% Cl 0.58-0.9). Inter-observer ICC value was moderate as well $-0.7(95 \% \mathrm{Cl} 0.43-0.86)$.

Table 1 shows clinical data after cut-off value usage to separate PD subjects with $\mathrm{SN} \leq 0.19 \mathrm{~cm}^{2}$ and $\mathrm{SN}>0.19 \mathrm{~cm}^{2}$. There was no difference between 
Table 1. Demographic and clinical data of Parkinson's disease patients

\begin{tabular}{lccc}
\hline & $\mathbf{S N}$ & $\mathbf{S N}$ & $\mathbf{P}$ \\
& $\leq \mathbf{0 . 1 9} \mathbf{c m}^{\mathbf{2}}$ & $\mathbf{>} \mathbf{0 . 1 9} \mathbf{~ m}^{2}$ & \\
\hline Number of patients & 17 & 78 & $\mathrm{NA}$ \\
Age [years] & $59 \pm 9$ & $62 \pm 11$ & 0.5 \\
Sex (male/female) & $8 / 9$ & $49 / 29$ & 0.3 \\
Disease duration [years] & $7 \pm 4$ & $7 \pm 2$ & 0.5 \\
MDS-UPDRS score & $28.5 \pm 12$ & $38.5 \pm 20$ & 0.2 \\
Hoehn and Yahr stage & $2.5 \pm 1$ & $2 \pm 0,5$ & 0.2 \\
Tremor dominant type & 2 & 14 & \\
Akinetic-rigid type & 3 & 26 & 0.4 \\
Mixed type & 10 & 40 & \\
\hline
\end{tabular}

subgroups in age, sex, disease duration, as well as MDS-USRS and Hoehn and Yahr stages of disease.

There was no statistically significant difference in the SN echogenic area between the TD, AR, and mixed type PD patients.

\section{DISCUSSION}

Consistent with number of previous reports from independent centres [14, 20, 21, 25, 28] our study confirmed a morphological abnormality of the SN visualised by TCS in the majority of PD patients. It is the first trial that investigated the size of SN hyperechogenic area in a large group of Polish population. We defined $\mathrm{SN}>0.19 \mathrm{~cm}^{2}$ as an abnormal extension of hyperechogenicity with the sensitivity is $86.3 \%$, specificity $94 \%$, with positive predictive value of $85.7 \%$, and negative predictive value of $82.8 \%$.

This study showed that SN hyperechogenicity in PD patients was not correlated with age, disease duration, Hoehn and Yahr staging or the unified PD rating scale motor symptoms, disease severity, that harmonised with most of the previous reports [25, 31]. We also confirmed earlier findings that the $\mathrm{SN}$ hyperechogenicity is not different among the motor subcategories of PD [11]. Therefore, all these data may suggest that structural changes revealed in TCS do not determine severity of PD symptoms.

We have also noticed that $10 \%$ of control subject had this ultrasound phenomenon, which lies within formerly reported $8-10 \%$ range.

Recent results of several longitudinal studies have shown that SN hyperechogenicty does not differ in size during the course of PD $[3,8]$. Finally the pro- spective study demonstrated a highly increased risk for PD in healthy elderly individuals with SN hyperechogenicity presence [10].

Hence, most of experts in this field treat this TCS phenomenon as a marker of PD, which can be helpful not only in early diagnosis but also in the predisposition assessment of this disorder [6]. There are several studies, which revealed the extent of SN hyperechogenicity in asymptomatic carriers of mutations of well-known PD-causing genes, as LRRK2 and PARK2 [12, 18].

However, there are 2 crucial points of criticism regarding $\mathrm{SN}$ area evaluation: the first one is a dependence on the examiner's experience. In our study intra-rater reliability in experienced neurosonographer was relatively good but inter-observer reliability (qualified vs. inexperienced sonographer in TCS) was moderate. This is consistent with previous reports that SN hyperechogenicity measurement reproducibility is high when performed by 1 experienced investigator $[26,27]$.

The second issue is the bias of the examiner who can notice evident extrapyramidal signs in patients with severe PD. In 1 recent interesting study sonographers were completely blinded: the examination room was completely darkened and the patients were veiled. Still the positive predictive value for PD was very high, i.e. $85.7 \%$ [24].

The basis of SN hyperechogenecity phenomenon is not clear enough. TCS of post-mortem brains and animal studies indicate that this abnormality may be related to iron deposition with formation of free radicals $[7,9]$.

There is also a hypothesis that it could be the result of microglial activation [5]. Further studies in this area are needed.

There is one important limitation of our work: it was carried on well-defined PD patients; we did not include any subjects with atypical Parkinsonian syndrome. However, the aim of the study was to estimate the presence of SN hyperechogenicity in clear condition in homogenous population of Polish patients. The role of SN abnormalities assessment in differential diagnostics might be analysed in our following studies.

\section{CONCLUSIONS}

In conclusion, SN hyperechogenicity as measured by TCS is a distinguishing feature related to PD that can improve the routine practice of movement disorders specialists. 


\section{REFERENCES}

1. Ambrosius W, Kozubski W (2010) Transcranial sonography in diagnosis of movement disorders. Neurol Neurochir Pol, 44: 574-579.

2. Becker G, Seufert J, Bogdahn U, Reichmann H, Reiners K (1995) Degeneration of substantia nigra in chronic Parkinson's disease visualized by transcranial color-coded real-time sonography. Neurology, 45: 182-184.

3. Behnke S, Runkel A, Kassar AS, Ortmann M, Guidez D, Dillmann U, Fassbender K, Spiegel J (2013) Long-term course of substantia nigra hyperechogenicity in Parkinson's disease. Mov Disord, 28: 455-459.

4. Berg D, Becker G, Zeiler B, Tucha O, Hofmann E, Preier M, Benz P, Jost W, Reiners K, Lange KW (1999) Vulnerability of the nigrostriatal system as detected by transcranial ultrasound. Neurology, 53: 1026-1031.

5. Berg D, Godau J, Riederer P, Gerlach M, Arzberger T (2010) Microglia activation is related to substantia nigra echogenicity. J Neural Transm, 117: 1287-1292.

6. Berg D, Godau J, Walter U (2008) Transcranial sonography in movement disorders. Lancet Neurol, 7: 1044-1055.

7. Berg D, Grote $C$, Rausch WD, Maeurer M, Wesemann W, Riederer P, Becker G (1999) Iron accumulation in the substantia nigra in rats visualized by ultrasound. Ultrasound Med Biol, 25: 901-904.

8. Berg D, Merz B, Reiners K, Naumann M, Becker G (2005) Five-year follow-up study of hyperechogenicity of the substantia nigra in Parkinson's disease. Mov Disord, 20: 383-385.

9. Berg $D$, Roggendorf W, Schröder U, Klein R, Tatschner T, Benz P, Tucha O, Preier M, Lange KW, Reiners K, Gerlach M, Becke G (2002) Echogenicity of the substantia nigra: association with increased iron content and marker for susceptibility to nigrostriatal injury. Arch Neurol, 59: 999-1005.

10. Berg D, Seppi K, Behnke S, Liepelt I, Schweitzer K, Stockner H, Wollenweber F, Gaenslen A, Mahlknecht P, Spiegel J, Godau J, Huber H, Srulijes K, KiechI S, Bentele M, Gasperi A, Schubert T, Hiry T, Probst M, Schneider V, Klenk J, Sawires M, Willeit J, Maetzler W, Fassbender K, Gasser T, Poewe W (2011) Enlarged substantia nigra hyperechogenicity and risk for Parkinson disease: a 37-month 3-center study of 1847 older persons. Arch Neurol, 68: 932-937.

11. Berg D, Siefker C, Becker G (2001) Echogenicity of the substantia nigra in Parkinson's disease and its relation to clinical findings. J Neurol, 248: 684-689.

12. Brüggemann N, Hagenah J, Stanley K, Klein Ch, Wang C, Raymond D, Ozelius L, Bressman S, Saunders-Pullman R (2011) Substantia nigra hyperechogenicity with LRRK2 G2019S mutations. Mov Disord, 26: 885-888.

13. Campenhausen S, Bornschein B, Wick R, Bötzel K, Sampaio C, Poewe W, Oertel W, Siebert U, Berger K, Dodel R (2005) Prevalence and incidence of Parkinson's disease in Europe. Eur Neuropsychopharmacol, 15: 473-490.

14. Doepp F, Plotkin M, Siegel L, Kivi A, Gruber D, Lobsien E, Kupsch A, Schreiber SJ (2008) Brain parenchyma sonography and 123I-FP-CIT SPECT in Parkinson's disease and essential tremor. Mov Disord, 23: 405-410.

15. Elbaz A, Bower JH, Maraganore DM, McDonnell SK, Peterson BJ, Ahlskog JE, Schaid DJ, Rocca W (2002) Risk tables for parkinsonism and Parkinson's disease. J Clin Epidemiol, 55: 25-31.

16. Gelb DJ, Oliver E, Gilman S (1999) Diagnostic criteria for Parkinson disease. Arch Neurol, 56: 33-39.
17. Goetz CG, Fahn S, Martinez-Martin P, Poewe W, Sampaio C, Stebbins GT, Stern MB, Tilley BC, Dodel R, Dubois B, Holloway R, Jankovic J, Kulisevsky J, Lang AE, Lees A, Leurgans S, LeWitt PA, Nyenhuis D, Olanow W, Rascol O, Schrag A, Teresi JA, Van Hilten JJ, LaPelle N (2007) Movement Disorder Society-sponsored revision of the Unified Parkinson's Disease Rating Scale (MDS-UPDRS): Process, format, and clinimetric testing plan. Mov Disord, 22: 41-47.

18. Hagenah JM, König IR, Becker B, Hilker R, Kasten $M$, Hedrich K, Pramstaller PP, Klein C, Seidel G. (2007) Substantia nigra hyperechogenicity correlates with clinical status and number of Parkin mutated alleles. J Neurol, 254: 1407-1013.

19. Hoehn MM, Yahr MD (1967) Parkinsonism: onset, progression, and mortality. Neurology, 17: 427-442.

20. Huang YW, Jeng JS, Tsai CF, Chen LL, Wu RM (2007) Transcranial imaging of substantia nigra hyperechogenicity in a Taiwanese cohort of Parkinson's disease. Mov Disord, 22: 550-555.

21. Kolevski G, Petrov I, Petrova V (2007) Transcranial sonography in the evaluation of Parkinson disease. J Ultrasound Med, 26: 509-512.

22. Korchounov A, Schipper HI, Preobrazhenskaya IS, Kessler KR, Yakhno NN (2004) Differences in age at onset and familial aggregation between clinical types of idiopathic Parkinson's disease. Mov Disord, 19: 1059-1064.

23. Lo RY, Tanner CM (2013) Epidemiology. In: Pahwa R, Lyons KE eds. Handbook of Parkinson's disease. 5 Ed. CRC Press, London, pp. 24-39.

24. Prestel J, Schweitzer KJ, Hofer A, Gasser T, Berg D (2006) Predictive value of transcranial sonography in the diagnosis of Parkinson's disease. Mov Disord, 21: 1763-1765.

25. Ressner P, Skoloudík D, Hlustík P, Kanovský P (2007) Hyperechogenicity of the substantia nigra in Parkinson's disease. J Neuroimaging, 17: 164-167.

26. Skoloudík $D$, Fadrná T, Bártová $P$, Langová $K$, Ressner $P$, Zapletalová O, Hlustík P, Herzig R, Kannovský P (2007) Reproducibility of sonographic measurement of the substantia nigra. Ultrasound Med Biol, 33: 1347-1352.

27. Van De Loo S, Walter U, Behnke S, Hagenah J, Lorenz M, Sitzer M, Hilker R, Berg D (2010) Reproducibility and diagnostic accuracy of substantia nigra sonography for the diagnosis of Parkinson's disease. J Neurol Neurosurg Psychiatry, 81: 1087-1092.

28. Vlaar AM, De Nijs T, Van Kroonenburgh MJ. Mess WH, Winogrodzka A, Tromp SC, Weber WE (2008) The predictive value of transcranial duplex sonography for the clinical diagnosis in undiagnosed parkinsonian syndromes: comparison with SPECT scans. BMC Neurology, 8: 42.

29. Walter U (2012) Transcranial sonography of the cerebral parenchyma: Update on clinically relevant applications. Perspectives in Medicine, 1: 334-343.

30. Walter U, Behnke S, Eyding J, Niehaus L, Postert T, Seidel G, Berg D (2007) Transcranial brain parenchyma sonography in movement disorders: state of the art. Ultrasound Med Biol, 33: 15-25.

31. Walter U, Dressler D, Wolters A, Wittstock M, Benecke R (2007) Transcranial brain sonography findings in clinical subgroups of idiopathic Parkinson's disease. Mov Disord, 22: 48-54.

32. Walter U, Wittstock M, Benecke R,Dressler D (2002) Substantia nigra echogenicity is normal in non-extrapyramidal cerebral disorders but increased in Parkinson's disease. J Neural Transm, 192: 191-196. 